\title{
The effect of boundary constraints on finite element modelling of the human pelvis
}

\author{
Peter J. Watson*, Ali Dostanpor, Michael J. Fagan and Catherine A. Dobson \\ Medical and Biological Engineering Research Group, School of Engineering and Computer Science, \\ University of Hull, Hull, HU6 7RX, UK
}

\begin{abstract}
The use of finite element analysis (FEA) to investigate the biomechanics of anatomical systems critically relies on the specification of physiologically representative boundary conditions. The biomechanics of the pelvis has been the specific focus of a number of FEA studies previously, but it is also a key aspect in other investigations of, for example, the hip joint or new design of hip prostheses. In those studies, the pelvis has been modelled in a number of ways with a variety of boundary conditions, ranging from a model of the whole pelvic girdle including soft tissue attachments to a model of an isolated hemi-pelvis. The current study constructed a series of FEA models of the same human pelvis to investigate the sensitivity of the predicted stress distributions to the type of boundary conditions applied, in particular to represent the sacro-iliac joint and pubic symphysis. Varying the method of modelling the sacro-iliac joint did not produce significant variations in the stress distribution, however changes to the modelling of the pubic symphysis were observed to have a greater effect on the results. Over-constraint of the symphysis prevented the bending of the pelvis about the greater sciatic notch, and underestimated high stresses within the ilium. However, permitting medio-lateral translation to mimic widening of the pelvis addressed this problem. These findings underline the importance of applying the appropriate boundary conditions to FEA models, and provide guidance on suitable methods of constraining the pelvis when, for example, scan data has not captured the full pelvic girdle. The results also suggest a valid method for performing hemi-pelvic modelling of cadaveric or archaeological remains which are either damaged or incomplete.
\end{abstract}

Key words: Biomechanics; finite element analysis; pelvis; musculoskeletal.

* Corresponding author. Address: Medical and Biological Engineering Research Group, School of Engineering and Computer Science, University of Hull, Hull, HU6 7RX, UK, Tel.: +44 1482 4666319, fax: +44 (0) 1482466664 E-mail: p.j.watson@hull.ac.uk 


\section{Introduction}

Total hip arthroplasty is considered one of the most successful orthopaedic interventions [1], and its importance is only set to increase with an increasingly aging population. This success has been aided by the continuous development of hip joint replacement designs [2], in terms of strength to support loads while limiting stress shielding to the surrounding bone [3], material choice [4] and method of fixation [5]. Evaluation of these design factors often utilises computational methods such as multi-body dynamics and musculoskeletal (MS) modelling to predict loading in the normal joint [6-8], and after prosthetic implantation [9]. This has been used in conjunction with finite element analysis (FEA) to estimate stress distributions in the femoral stem [10,11] and acetabular cup [12], wear rates [13] and to assess bone remodelling after implantation [14]. However, to evaluate such factors it is important to understand the force transfer through the hip joint during normal loading conditions.

Modelling of the hip joint must consider the physiology of the pelvis and its associated joints. The pelvis articulates with the femur through the hip joint, and the sacrum via the sacro-iliac joint (SIJ), while the pubic symphysis connects the two hemi-pelves. The hip joint is reported to be a generalised ball-andsocket articulation joint [15] and studies have investigated the biomechanics of the pubic symphysis [16], but less is known about the interaction of the SIJ. The SIJ permits movement in both rotation and translation [17], although there is a ligamentous structure that limits this motion and stabilises the joint [18]. The SIJ is an important link that facilitates the transfer of upper body weight through to the lower limbs. Therefore it is important to model these joints accurately in multi-body dynamic and FEA modelling, in order to accurately predict load transfers and the associated stress distributions.

Previous FEA models of the pelvis vary in complexity and the approaches used to capture the movement at the joints frequently differ between studies. Some studies have taken advantage of the symmetrical nature of the structure and only model the hemi-pelvis, with the SIJ and pubic symphysis constrained in all degrees of freedom (DOF) [19-21]. Coultrup et al. [22] also modelled the pelvis in a simailar manner but with symmetric conditions defined at the pubic symphysis. Attempts to simplify modelling of the pelvic girdle have been made by eliminating the sacrum and constraining the both SIJs in all DOF $[23,24]$. In contrast, all of the bones within the pelvic girdle have also been modelled, enabling the pelvis to freely rotate about the sacrum, while connecting the two hemi-pelves through the inter-pubic disc $[25,26]$.

The sensitivity of material properties [27,28] and boundary conditions [25,29-31] considered in FEA of the pelvis has been previously investigated. Phillips et al. [25] and Hao et al. [29] both reported a variation in stress distributions between modelling a constrained SIJ and inclusion of a ligamentous structure. In contrast, Ghosh et al. [30] examined the effect of enabling varying degrees of movement at the pubic symphysis, and found no difference within the stresses of the lateral cortex, but variations 
were observed medially. However, none of these previous studies examined the sensitivity of simultaneously varying the boundary conditions of both joints. Clarke et al [31] did model the pelvic girdle with ligaments spanning the SIJ and a hemi-pelvis with fixed constraints at the SIJ and pubic symphysis, reporting no significant difference in peak stresses and strains. However, this study was limited in its analysis, considering only the stresses and strains at the acetabulum, and therefore did not investigate the effect on other areas of the pelvis.

This study initially considered an FEA model of the pelvic girdle to predict the stress and strain distributions associated with MS loading. The complexity of this model was subsequently reduced via the application of constraints at the SIJ and pubic symphysis, and the resulting variation in the stress and strain distributions were analysed. This aimed to assess the level to which the pelvic anatomy can be simplified in FEA modelling before the predicted stresses and strains within the pelvis become incomparable.

\section{Material and methods}

\subsection{Musculoskeletal modelling}

The forces experienced by the pelvis during walking were predicted using an existing MS model which is freely available from the AnyBody Managed Model Repository v.1.3.1 (AnyBody Technology, Aalborg, Denmark). The model has a detailed representation of the lower extremity, incorporating full muscle wrapping and containing numerous strands for large pennate muscles (e.g. the glutei and iliacus), in order to capture numerous lines of action. The model is driven by three-dimensional (3D) motion capture data, which includes accompanying ground force reactions, of two successive gait cycles (heel strike-heel strike). Simulations were performed in the AnyBody Modelling System v.5.0, which utilises inverse dynamics to compute muscle and joint forces associated with the motion capture data.

A single gait cycle was then analysed which consisted of a left-legged stance phase, and the forces of 22 muscles spanning the hip joint were recorded for the left side. The accompanying resultants of the hip joint reaction force were also recorded. Two load regimes were subsequently created which corresponded to phases of the gait cycle containing the largest hip joint reactions, in this case: at initial heel strike ( $\sim 15 \%$ of the gait cycle); and, just before toe-off ( $\sim 48 \%$ of the gait cycle). Hereafter these load regimes are referred to as the $15 \%$ loading regime and $48 \%$ loading regime.

\subsection{Finite element modelling}

The CT dataset of the male visible human [32] was digitised in AVIZO image visualization software v.6.3 (Visualization Sciences Group, Burlington, MA, USA) and segmented to create a solid 3D volumetric model which comprised of the two hemi-pelvic bones, sacrum, SIJ cartilage and inter-pubic disc. This volumetric model was meshed within AVIZO with 10-node tetrahedral elements. The mesh 
was subsequently imported to ANSYS v.14.5 (ANSYS Inc., Canonsburg, PA, USA), where 6-node triangular shell elements were clad around the pelvic bones to represent the cortical bone. In total, the model of the pelvic girdle consisted of 389,795 elements $(385,924$ representing bone and 3,871 representing cartilaginous material).

Five separate FEA models were constructed to investigate the effects of different boundary conditions, and the validity of modelling only the hemi-pelvis in comparison to the full pelvic girdle (see Error! Reference source not found.). The models were a gradual simplification of the most complex scenario (Model 1) which contained the pelvic girdle and constrained nodes located on the superior surface of the sacrum in all DOF (see Error! Reference source not found.(a)). This was simplified by eliminating the sacrum to create: Model 2 where 10 nodes around the circumference of the exterior surface of the SIJ were constrained in all DOF; and Model 3 where the cartilage was eliminated and the exterior nodes of the pelvic bone within the SIJ articulation area were constrained in all DOF (see Error! Reference source not found.(b)). This allowed the influence of pelvic rotation about the SIJ to be examined. The pelvis was then further simplified by eliminating the right hemi-pelvis and connecting cartilaginous material, and creating two left hemi-pelvic models: one with the exterior nodes of the pelvic bone in the SIJ articulation region constrained in all DOF and the exterior nodes at the pubic symphysis permitted to move in medio-lateral translation only (in order to simulate the natural widening of the pelvis) (Model 4); and another, with the exterior nodes of the pelvic bone in the SIJ articulation region and pubic symphysis constrained in all DOF (Model 5) (see Error! Reference source not found.(c)).

The areas of origin for the 22 pelvic muscles areas were mapped onto the mesh of the left-hemi-pelvis, with the larger muscles divided into sections (i.e. anterior, mid-section, posterior) in order to replicate the differing lines of action captured by the MS model. For muscles with a single line of action, the predicted muscle force of the MS model was divided by the total number of nodes within the origin site. Using the line of action, this distributed force was then resolved into its resultant forces (i.e. Fx, Fy and Fz), which were subsequently applied to the nodes within the origin site. The same method was used to define the larger pennate muscles, although the forces along the separate lines of action were distributed over the corresponding divided section (i.e. anterior section etc). The MS model also represents muscles which wrap around bony prominences and other soft tissues, using one or more "via points" between the origin and insertion. This wrapping was replicated on the left hemi-pelvis through applying a single line of action between the muscle origin and the nearest "via point". The hip joint reaction force was applied as a distributed load over the nodes within the acetabulum. Since the MS and FEA models were based on different subjects, the muscle and joint forces were scaled to the size of the male visible human pelvis using a ratio between the pelvic width (measured as the distance between the two anterior superior iliac spines (ASIS)) and pelvic depth (measured as the distance between the ASIS and the posterior superior iliac spine (PSIS)). A ratio of these two measures was taken as they are both 
considered when scaling a patient-specific MS model to motion capture data. This resulted in the MS forces being scaled by a factor of 1.14 before being applied to the FE model. Thus the MS forces associated with the $15 \%$ and $48 \%$ loading regimes were applied to the separate models in this way. As the same mesh was used in each model, the locations of the muscle and hip joint reaction forces were of course identical in each case.

The trabecular and cortical bone were modelled as linear, elastic and isotropic, with properties taken from the literature of previous pelvic FEA studies. Thus the Young's modulus (E) of the trabecular bone was assumed to be 70MPa (with $v=0.2$ ) and that of the cortical bone was $17 \mathrm{GPa}$ (with $v=0.3$ ) $[24,27]$. Unfortunately, the thickness of the pelvic cortical bone could not be determined from the CT data, however Anderson et al. [28] reported that it ranges between $0.44-4.00 \mathrm{~mm}$ (average $=1.41 \mathrm{~mm}$ ), therefore a homogeneous value of $1.5 \mathrm{~mm}$ was applied. The SIJ cartilage and inter-pubic disc were defined as a three-parameter Mooney-Rivlin model, with coefficients of $\mathrm{C}_{10}=0.1 \mathrm{MPa}, \mathrm{C}_{01}=0.45 \mathrm{MPa}$ and $\mathrm{C}_{11}=0.6 \mathrm{MPa}$ [33]. Since this study was focused on the sensitivity of stresses within the pelvis, the sacrum was simply defined with the properties of cortical bone. In order to enable rotation of the pelvis about the SIJ cartilage in Model 2, the soft tissue was modelled as a linear, elastic material with the properties of cortical bone.

\section{Results}

To investigate the effect of boundary conditions upon pelvic biomechanics, the predicted deformation, von Mises stress, third principal (compressive) strain and first principal (tensile) strain distributions were compared between the five models. Each model was analysed during both the $15 \%$ and $48 \%$ loading regimes, with the predicted displacement and stress and strain distributions compared in the cortical bone of the left hemi-pelvis.

As shown in Error! Reference source not found., the greatest displacement was produced in Model 1 , with the ischial-pubic structure displacing by up to an order of magnitude greater than that observed with the other models. A large section of the iliac crest which extended to the lesser sciatic notch, also displayed by similar magnitudes under the $48 \%$ loading regime (Error! Reference source not found.(a)). Displacement significantly reduced in Models 2 and 3, with only small regions around the ischial tuberosity and ASIS displacing by the large magnitudes previously observed (Error! Reference source not found.(b) \& (c)). Despite enabling rotation of the pelvis in Model 2, there were only minor differences between displacements in Models 2 and 3 when the $15 \%$ loading regime was applied. However, greater variation was observed under the $48 \%$ loading regime, with Model 2 displaying larger movement of the iliac crest near the ASIS and within the inferior region of the ischial tuberosity. A further reduction in displacement was observed when only the hemi-pelvis was modelled. Although Model 4 produced less movement along the ilio-pubic line and the pubic symphysis than any preceding model under the $15 \%$ loading regime, these regions were more mobile when compared with Model 3 
during the $48 \%$ loading regime (Error! Reference source not found.(d)). In comparison, Model 5 produced the lowest displacements in comparison to the other models under both loading regimes, with the majority of the structure experiencing minimal movement during the $48 \%$ loading regime (Error! Reference source not found.(e)).

When comparing the von Mises stresses, the different models were found to have similar distributions (Error! Reference source not found.). These were primarily characterised on the lateral side by a high stress concentration in the inferior ilium and along the superior regions of the acetabular rim. These high stresses were observed to move towards the posterior inferior iliac spine under the $15 \%$ loading regime, producing a high concentration covering the majority of the inferior ilium. High stresses concentrated medially around the SIJ and extended along the ilio-pubic line. Stresses predicted during the 15\% loading regime remained remarkably consistent between Models 1-3 (Error! Reference source not found.(a) \& (c)). A similar observation was also made with the $48 \%$ loading regime, although there was slight stress relief in the medial body of the pubis in Model 3. In comparison, a greater variation was observed within the hemi-pelvic modelling, as Model 4 displayed larger stresses in the medial body of the ischium but stress relief around the SIJ, under the 15\% loading regime (Error! Reference source not found.(d)). There was a further stress increase throughout the majority of the ischial-pubic structure under the $48 \%$ loading regime, although the distribution in the ilium was similar to the preceding models. A noticeable stress reduction was observed around the SIJ within Model 5, which is most evident during the $48 \%$ loading regime (Error! Reference source not found.(e)). This was accompanied by an increase in stress around the pubic symphysis.

Similar observations were found with compressive strains, with Models 1-3 displaying large strain concentrations within the inferior and posterior ilium laterally, and around the SIJ medially, under the 15\% loading regime (Error! Reference source not found.). These models also exhibited similar distributions under the $48 \%$ loading regime, which was characterised by higher strains around the SIJ and inferior ilium medially, but with strain relief around the PSIS laterally. Once again, a variation was observed within Model 4 where the 15\% loading regime produced slight strain relief around the SIJ medially, although this was accompanied by a larger concentration of high strain within the lateral inferior ilium. In contrast, Model 5 displayed noticeably lower strains around the SIJ and increased strains around the pubic symphysis, for both loading regimes.

Fig. 5 illustrates the distribution of tensile strains was similar between Models 1-3 for both loading regimes. In comparison, strain relief was observed around the SIJ within Models 4 and 5 (with the exception of Model 4 under the $48 \%$ loading regime), while larger tensile strains concentrated within the lateral body of the pubis and around the pubic symphysis. 
To more easily quantify the sensitivity of the predicted von Mises stresses within the ischial-pubic structure, the nodal stresses at two locations on the surface of the pubis and ischium (one medial and one lateral) (Fig. 6) were recorded during the two loading regimes. To eliminate possible anomalies associated with analysing single nodes, an averaged stress was calculated from typically 7 nodes that surrounded the selected locations. The same nodes were analysed in each model. The nodal stresses within each model were subsequently analysed as a percentage error in comparison to Model 1. Under the $15 \%$ loading regime it was observed that Models 2 and 3 produced a maximum variation of up to $45 \%$ within the pubis (Fig. 6 (a)). While this is a sizeable difference, it is modest considering the much larger variation observed within Model 5, which produced a $200 \%$ increase on the medial side. In comparison, the errors in the ischial stresses were significantly reduced, with Model 4 producing a maximum variation of 33\% (Fig. 6 (b)). Analysis of the $48 \%$ loading regime illustrates that pubic and ischial stresses within Model 2 were similar to those of Model 1 (Fig. 7). A larger difference was observed within Model 3, although the maximal error in each bone was lower when compared to the previous loading regime. Once again, the hemi-pelvic models produced the largest deviations from the baseline model, with maximum errors of 57\% (Model 4) and 77\% (Model 5) in the pubis and ischium, respectively (Fig. 7).

Finally, to visualise the differences between the modelling of the pelvic girdle and hemi-pelvis, the element von Mises stresses in Model 1 were subtracted from those in Model 5, when loaded with the $15 \%$ loading regime. The resulting stress plots show that Model 1 had larger stress concentrations throughout the majority of the ilium, particularly around the SIJ (Fig. 8 (a)). In comparison, Model 5 had higher stresses that mainly pertained to the pubis, and the ilio-ischial scar on the medial side (Fig. $8(b))$.

\section{Discussion}

Design and evaluation of hip joint replacement designs often utilise FEA modelling of the pelvis $[14,22,34]$. This requires knowledge of the best way to model the pelvic joints in order to represent the in vivo conditions. Previous FEA studies have employed a range of different methods to replicate the physiological constraints of the pelvis, ranging from hemi-pelvic modelling [19,21,22,34] to simulations using the complete pelvic girdle $[25,26]$. This study has investigated the effects of simplifications in the representation of the physiological constraints, upon the biomechanical response of the pelvis during musculoskeletal loading.

Modelling of the complete pelvic girdle (Model 1) produced the greatest displacements during loading, particularly along the iliac crest, ischium and pubis (Error! Reference source not found.(a)). Eliminating the sacrum and constraining the SIJ directly caused a significant reduction in overall displacement, although only minor differences were observed where rotation was permitted compared 
to the case where joint movement was restricted in all DOF (Error! Reference source not found.(b) $\&(c))$. Despite these variations in overall displacements, the von Mises stress, compressive strain and tensile strain distributions predicted by Models 1-3 were similar (Error! Reference source not found.(a)-(c), Error! Reference source not found.(a)-(c), Fig. 5(a)-(c), Fig. 6 and Fig. 7). This occurs because both loadings regimes cause the whole pelvic structure (in all three modelling scenarios) to move in a superio-lateral direction. Therefore, because the pelvis generally maintains its physiological shape with both hemi-pelves moving in unison rather than the separate displacement of the left hemipelvis, the predicted stress distributions are similar.

Hemi-pelvic modelling produced less movement of the pubic symphysis, with the exception of Model 4 under the $48 \%$ loading regime (Error! Reference source not found.(d) \& (e)). Through enabling medio-lateral movement of the pubic symphysis in Model 4, greater movement of the ilio-pubic structure was produced in comparison with Model 5. Consequently, Model 4 displaced in a lateral direction (similar to the preceding models) although Model 5 deformed in a manner not previously observed. The resulting stress and strain distributions generated higher values around the pubic symphysis and significant stress/strain relief around the SIJ, with the exception of Model 4 under the 48\% loading regime (Error! Reference source not found.(d) \& (e), Error! Reference source not found.(d) \& (e) and Fig. 5(d) \& (e)). Furthermore, Fig. 6 and Fig. 7 illustrate that the hemi-pelvic modelling produced the largest variation in nodal von Mises stresses analysed in the ischial-pubic structure (with the exception of the ischium in Model 5 under the 15\% loading regime). This is due to the pubic symphysis movement in Models 1-3 causing bending of the hemi-pelvis around the greater sciatic notch. Consequently, high stresses and principal strains concentrate around this area and along the ilio-pubic line. Once movement of the pubic symphysis is restricted (as in Models $4 \& 5$ ), this bending is significantly reduced and results in stress and strain relief. This effect is clearly visible in Fig. 8, where Model 1 has higher stresses throughout the majority of the ilium, whereas Model 5 has higher stresses within the pubis.

As far as the authors are aware, this is the first attempt to test the boundary conditions of both the SIJ and pubic symphysis in FEA models of the pelvis, and investigate the effects on the whole structure when using the same loading conditions. Although previous studies have analysed the different constraints within pelvic FEA modelling [25,29,31], either they only reported the effect within a localised area, or the loading was inconsistent between each model. Consequently, it is difficult to perform a direct comparison between the results of this study and those within literature. However, similarities can be observed; for example, Phillips et al. [25] also reported an alteration in von Mises stress distribution between pelvic girdle and hemi-pelvic modelling, albeit with different loading regimes in each case. Furthermore, the stress variations observed between Models 4 and 5 (Error! Reference source not found.) matched the findings of Clarke et al. [31], who reported consistent stresses laterally but altered distributions medially, with varying constraints at the pubic symphysis. 
Comparisons can also be made with previous pelvic FEA modelling which applied musculoskeletal loading associated with the gait cycle. For instance, Dalstra et al. [23] stated that hip joint loading was transferred through the cortex, from the acetabulum towards the SIJ and pubic symphysis. Therefore, high stress concentrations were reported within these regions, which is consistent with the observations of Error! Reference source not found. The average cortical stress magnitude during the two phases of the gait cycle model here was found to be in the range of 6.7-9.3MPa, which also agrees with values reported in the literature (e.g. 5-15MPa [24] and 15-20MPa [23]). Majumder et al. [24] found maximum displacements under such loading to be in the range $0.8-1.3 \mathrm{~mm}$, suggesting the displacements observed in Error! Reference source not found. are not physiologically unreasonable. In addition, the sample principal strains presented within Error! Reference source not found. and Fig. 5 are not excessive considering the physiological strains required for homeostasis. However, it is noted that the similarity in stresses around the SIJ between Models 1-3 is not consistent with the findings of Hao et al. [29], who reported variations in distributions when the SIJ was modelled in different ways. However, Hao et al. [29] modelled the connection between the pelvis and sacrum solely through ligaments, and unlike this study, did not include SIJ cartilage. Direct validation of the results of this current study is difficult. Overall, comparisons with previous investigations suggests that the distribution and magnitudes of the predicted von Mises stress and principal strains, are reasonable for gait related musculoskeletal loading, and therefore the relative differences in the results for the different boundary condition models can be accepted with confidence.

The use of FEA to investigate the biomechanics of anatomical systems relies on the specification of physiologically representative boundary conditions. Many studies consider the loading applied to the models with great care, but often pay little attention to the external constraints or internal connections between the different elements of the problem. Patient-specific models of the pelvic girdle are a good case in point, and should include both ligamentous and cartilage structures, requiring the use of CT data of the whole pelvic girdle with clear definition between bone and soft tissue. However, scan data does not always clearly capture such features, thus simplifications have to be considered. This study has shown that although different methods of modelling the pelvic girdle (i.e. with or without the sacrum) result in different magnitudes of displacement, the overall deformed shape is the same and similar stress distributions are generated. Therefore, although modelling the pelvic girdle should be considered the "gold standard" when analysing pelvic mechanics under MS loading, the inclusion of cartilaginous material at the SIJ may not influence the predicted results significantly, and the model constraints can be applied directly to the ilium.

Furthermore, to minimise radiation exposure, clinical CT scans often focus on a specific location and do not capture the whole pelvic girdle. In such instances it may be necessary to simplify the structure to a single hemi-pelvis (usually with no soft tissue). This study has demonstrated that hemi-pelvic modelling can produce comparative results to pelvic girdle modelling, providing that medio-lateral 
translation is permitted at the pubic symphysis to mimic widening of the inter-pubic disc. The validity of hemi-pelvic modelling is also advantageous when considering the increased time and computational power required for pelvic girdle modelling. This evidence also increases the feasibility and reliability of modelling archaeological remains, which do not contain any soft tissue and are often damaged or disarticulated.

The research, especially Error! Reference source not found., also highlights the varying stress states experienced by the pelvis due to loading from the gait cycle. Although both loading regimes have the largest concentrations located around the SIJ medially and within the inferior ilium laterally, differences could be seen in other regions. Largely due to activation of different muscle groups at the two phases of the gait cycle modelled, the $48 \%$ loading regime generally produced the higher stresses.

Despite detailed consideration of the boundary conditions in this study, inevitably there are other simplifications and limitations in the FEA models. Firstly, the effect of ligaments on the pelvic mechanics was not considered, particularly the sacro-iliac ligament which would have added further stability to the SIJ in Model 1, especially in response to higher loads. However, the displacements shown in Error! Reference source not found. were similar when compared to other studies which modelled gait loading. Unfortunately material properties for trabecular and cortical bone could not be accurately ascertained from the scan data, therefore homogeneous values from previous studies were used [24,27]. However, sensitivity studies of pelvic FEA models have shown that using homogeneous properties produces similar results when compared to modelling with heterogeneous properties [27,28]. In addition, different subjects were used in the MS and FEA modelling, requiring the use of a scaling factor to ensure the predicted forces were appropriate for the size of the male pelvis from the Visible Human Project. However, as this study aimed to assess the sensitivity of boundary constraints on the pelvis, rather than prediction of the true physiological stress/strain distributions during gait loading, these limitations were considered acceptable as they were the same for all models. Consequently, they are unlikely to have affected the observations and conclusions made.

This study has demonstrated that the application of boundary conditions requires careful consideration in the analysis of the pelvis. Modelling of the full pelvic girdle should be attempted if possible, in order to ensure accurate prediction of stress/strain distributions. However, in instances when this is not feasible, constraints applied directly to the SIJ have been shown to produce comparative results. Simplification of boundary conditions is more sensitive to the representation of the pubic symphysis compared to the SIJ, with over-constraint preventing the natural bending of the pelvis about the greater sciatic notch, thus not reflecting the high stresses that can occur round the SIJ and along the ilio-pubic line. This can be mediated through permitting medio-lateral translation to mimic widening of the pelvis, suggesting a valid method for performing hemi-pelvic modelling of cadaveric or archaeological remains which are either damaged or incomplete. These findings are useful in both the analysis of physiological 
stresses associated with normal daily activities, such as walking, but also more clinical applications, such alteration to bone remodelling after implantation of prosthetics.

\section{Conflicts of interest}

None declared.

\section{Competing interests}

None declared.

\section{Funding}

None.

\section{Ethical approval}

No required.

\section{References}

[1] Knight SR, Aujla R, Biswas SP. Total Hip Arthroplasty - over 100 years of operative history. Orthop Rev (Pavia) 2011;3:e16.

[2] Pramanik S, Agarwal AK, Rai KN. Chronology of total hip joint replacement and materials development. Trends Biomater Artif Organs 2005;19:15-26.

[3] Ridzwan MIZ, Shuib S, Hassan AY, Shokri AA, Mohammad Ibrahim MN. Problem of stress shielding and improvement to the hip implant designs: A review. J Med Sci 2007;7:460-7.

[4] Macdonald N, Bankes M. Ceramic on ceramic hip prostheses: A review of past and modern materials. Arch Orthop Trauma Surg 2014;134:1325-33.

[5] Abdulkarim A, Ellanti P, Motterlini N, Fahey T, O’Byrne JM. Cemented versus uncemented fixation in total hip replacement: a systematic review and meta-analysis of randomized controlled trials. Orthop Rev (Pavia) 2013;5:e8.

[6] Bergmann G, Deuretzbacher G, Heller M, Graichen F, Rohlmann A, Strauss J, et al. Hip contact forces and gait patterns from routine activities. J Biomech 2001;34:859-71.

[7] Hashimoto N, Ando M, Yayama T, Uchida K, Kobayashi S, Negoro K, et al. Dynamic analysis of the resultant force acting on the hip joint during level walking. Artif Organs 2005;29:387-92.

[8] Modenese L, Phillips ATM. Prediction of hip contact forces and muscle activations during walking at different speeds. Multibody Syst Dyn 2012;28:157-68.

[9] Zhang X, Chen Z, Wang L, Yang W, Li D, Jin Z. Prediction of hip joint load and translation using musculoskeletal modelling with force-dependent kinematics and experimental validation. Proc Inst Mech Eng Part H J Eng Med 2015;229:477-90.

[10] Abdullah AH, Asri MNM, Alias MS, Giha T. Finite element analysis of cemented hip arthroplasty: Influence of stem tapers. Proc. Int. MultiConference Eng. Comput. Sci. 2010, IMECS 2010:2241-6.

[11] Baharuddin MY, Salleh SH, Zulkifly AH, Lee MH, Noor AM, A Harris AR, et al. Design process of cementless femoral stem using a nonlinear three dimensional finite element analysis. BMC Musculoskelet Disord 2014;15.

[12] Mak M, Jin Z, Fisher J, Stewart TD. Influence of Acetabular Cup Rim Design on the Contact 
Stress During Edge Loading in Ceramic-on-Ceramic Hip Prostheses. J Arthroplasty 2011;26:131-6.

[13] Queiroz RD, Oliveira ALL, Trigo FC, Lopes JA. A finite element method approach to compare the wear of acetabular cups in polyethylene according to their lateral tilt in relation to the coronal plane. Wear 2013;298-299:8-13.

[14] Nie Y, Pei F, Li Z. Finite Element Modelling for Assessing Effect of Acetabular Component Orientation on the Basic Stress Path above Acetabular Dome. Orthop Surg 2015;7:66-73.

[15] Cereatti A, Margheritini F, Donati M, Cappozzo A. Is the human acetabulofemoral joint spherical? J Bone Jt Surg - Ser B 2010;92:311-4.

[16] Li Z, Alonso JE, Kim JE, Davidson JS, Etheridge BS, Eberhardt AW. Three-dimensional finite element models of the human pubic symphysis with viscohyperelastic soft tissues. Ann Biomed Eng 2006;34:1452-62.

[17] Goode A, Hegedus EJ, Sizer Jr P, Brismee JM, Linberg A, Cook CE. Three-dimensional movements of the sacroiliac joint: A systematic review of the literature and assessment of clinical utility. J Man Manip Ther 2008;16:25-38.

[18] Cohen SP, Chen Y, Neufeld NJ. Sacroiliac joint pain: A comprehensive review of epidemiology, diagnosis and treatment. Expert Rev Neurother 2013;13:99-116.

[19] Kaku N, Tsumura H, Taira H, Sawatari T, Torisu T. Biomechanical study of load transfer of the pubic ramus due to pelvic inclination after hip joint surgery using a three-dimensional finite element model. J Orthop Sci 2004;9:264-9.

[20] Cilingir AC, Ucar V, Kazan R. Three-dimensional anatomic finite element modelling of hemiarthroplasty of human hip joint. Trends Biomater Artif Organs 2007;21:63-72.

[21] Li J, Stewart TD, Jin Z, Wilcox RK, Fisher J. The influence of size, clearance, cartilage properties, thickness and hemiarthroplasty on the contact mechanics of the hip joint with biphasic layers. J Biomech 2013;46:1641-7.

[22] Coultrup OJ, Hunt C, Wroblewski BM, Taylor M. Computational assessment of the effect of polyethylene wear rate, mantle thickness, and porosity on the mechanical failure of the acetabular cement mantle. J Orthop Res 2010;28:565-70.

[23] Dalstra M, Huiskes R. Load transfer across the pelvic bone. J Biomech 1995;28:715-24.

[24] Majumder S, Roychowdhury A, Pal S. Biomechanical analysis of human pelvis under musculo-skeletal load using 3d finite element. Int J Appl Mech Eng 2005;10:646-7.

[25] Phillips ATM, Pankaj P, Howie CR, Usmani AS, Simpson AHRW. Finite element modelling of the pelvis: Inclusion of muscular and ligamentous boundary conditions. Med Eng Phys 2007;29:739-48.

[26] Shi D, Wang F, Wang D, Li X, Wang Q. 3-D finite element analysis of the influence of synovial condition in sacroiliac joint on the load transmission in human pelvic system. Med Eng Phys 2014;36:745-53.

[27] Dalstra M, Huiskes R, Van Erning L. Development and validation of a three-dimensional finite element model of the pelvic bone. J Biomech Eng 1995;117:272-8.

[28] Anderson AE, Peters CL, Tuttle BD, Weiss JA. Subject-specific finite element model of the pelvis: Development, validation and sensitivity studies. J Biomech Eng 2005;127:364-73.

[29] Hao Z, Wan C, Gao X, Ji T. The effect of boundary condition on the biomechanics of a human pelvic joint under an axial compressive load: a three-dimensional finite element model. $\mathrm{J}$ Biomech Eng 2011;133:101006.

[30] Ghosh R, Pal B, Ghosh D, Gupta S. Finite element analysis of a hemi-pelvis: the effect of inclusion of cartilage layer on acetabular stresses and strain. Comput Methods Biomech Biomed Engin 2015;18:697-710. 
[31] Clarke SG, Phillips ATM, Bull AMJ. Evaluating a suitable level of model complexity for finite element analysis of the intact acetabulum. Comput Methods Biomech Biomed Engin 2013;16:717-24.

[32] Spitzer VM, Whitlock DG. The visible human dataset: The anatomical platform for human simulation. Anat Rec 1998;253:49-57.

[33] Leung ASO, Gordon LM, Skrinskas T, Szwedowski T, Whyne CM. Effects of bone density alterations on strain patterns in the pelvis: Application of a finite element model. Proc Inst Mech Eng Part H J Eng Med 2009;223:965-79.

[34] Cilingir AC, Ucar V, Kazan R. Three-dimensional anatomic finite element modelling of hemiarthroplasty of human hip joint. Trends Biomater Artif Organs 2007;21:63-72. 


\begin{tabular}{|c|c|}
\hline Model & Boundary condition \\
\hline 1 & Pelvic girdle with constraint applied at the sacrum \\
\hline 2 & $\begin{array}{l}\text { Pelvic girdle (minus sacrum) with nodes around the circumference of the } \\
\text { exterior surface of the SIJ constrained in all DOF }\end{array}$ \\
\hline 3 & $\begin{array}{l}\text { Pelvic girdle (minus sacrum and SIJ cartilage) with exterior nodes of the } \\
\text { pelvic bone within the SIJ articulation area constrained in all DOF }\end{array}$ \\
\hline 4 & $\begin{array}{l}\text { Left hemi-pelvis with the SIJ constrained in all DOF, and the pubic } \\
\text { symphysis allowed to translate in a medio-lateral direction }\end{array}$ \\
\hline 5 & Left hemi-pelvis with the SIJ and pubic symphysis constrained in all DOF \\
\hline
\end{tabular}

Table 1. Description of the boundary conditions applied to the FEA models. 


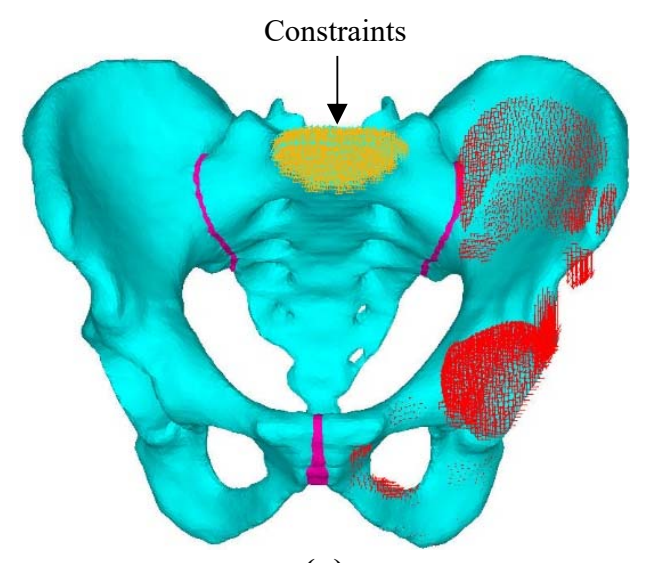

(a)

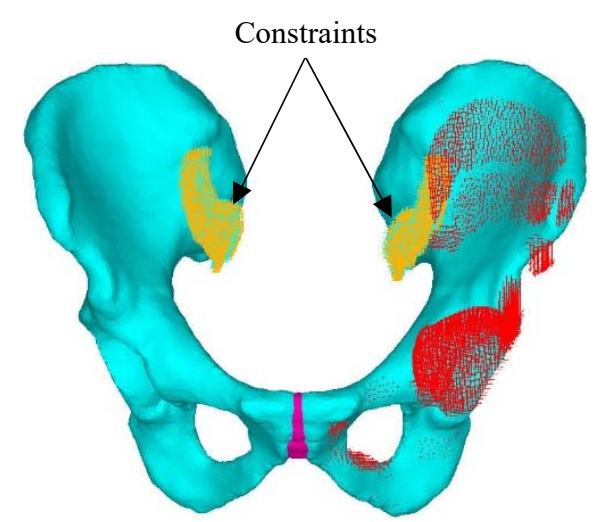

(b)

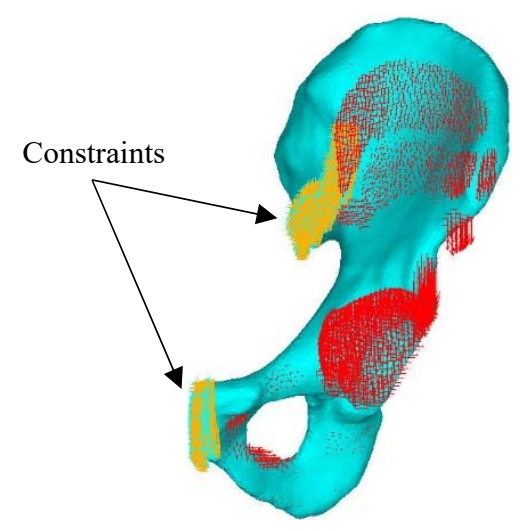

(c)

Fig. 1. FEA modelling of the pelvis with different boundary conditions, showing: (a) Model 1 - the pelvic girdle with nodes at the superior surface of the sacrum fixed in all DOF; (b) Model 3 - the pelvic girdle, minus the sacrum and SIJ cartilage, with the exterior nodes of the pelvic bone within the SIJ articulation area constrained in all DOF; and (c) Model 5 - the left hemi-pelvis with nodes at the SIJ and pubic symphysis constrained in all DOF. Models 2 and 4 are not shown because they are visually similar to Models 3 and 5 respectively. The loading indicated by the red arrows was identical in all models. 
(a)

(b)

(c)

(d)

(e)
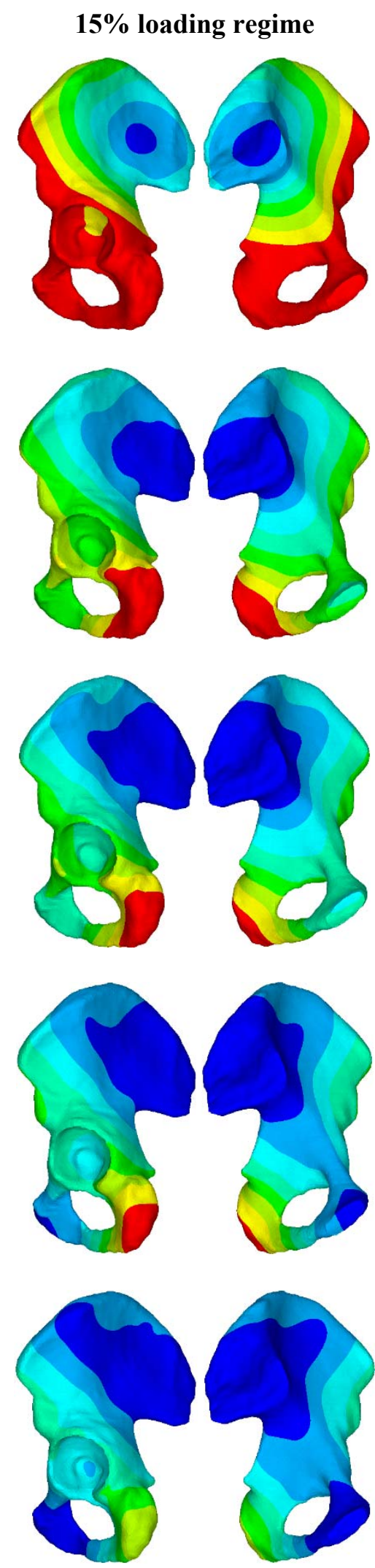

$48 \%$ loading regime
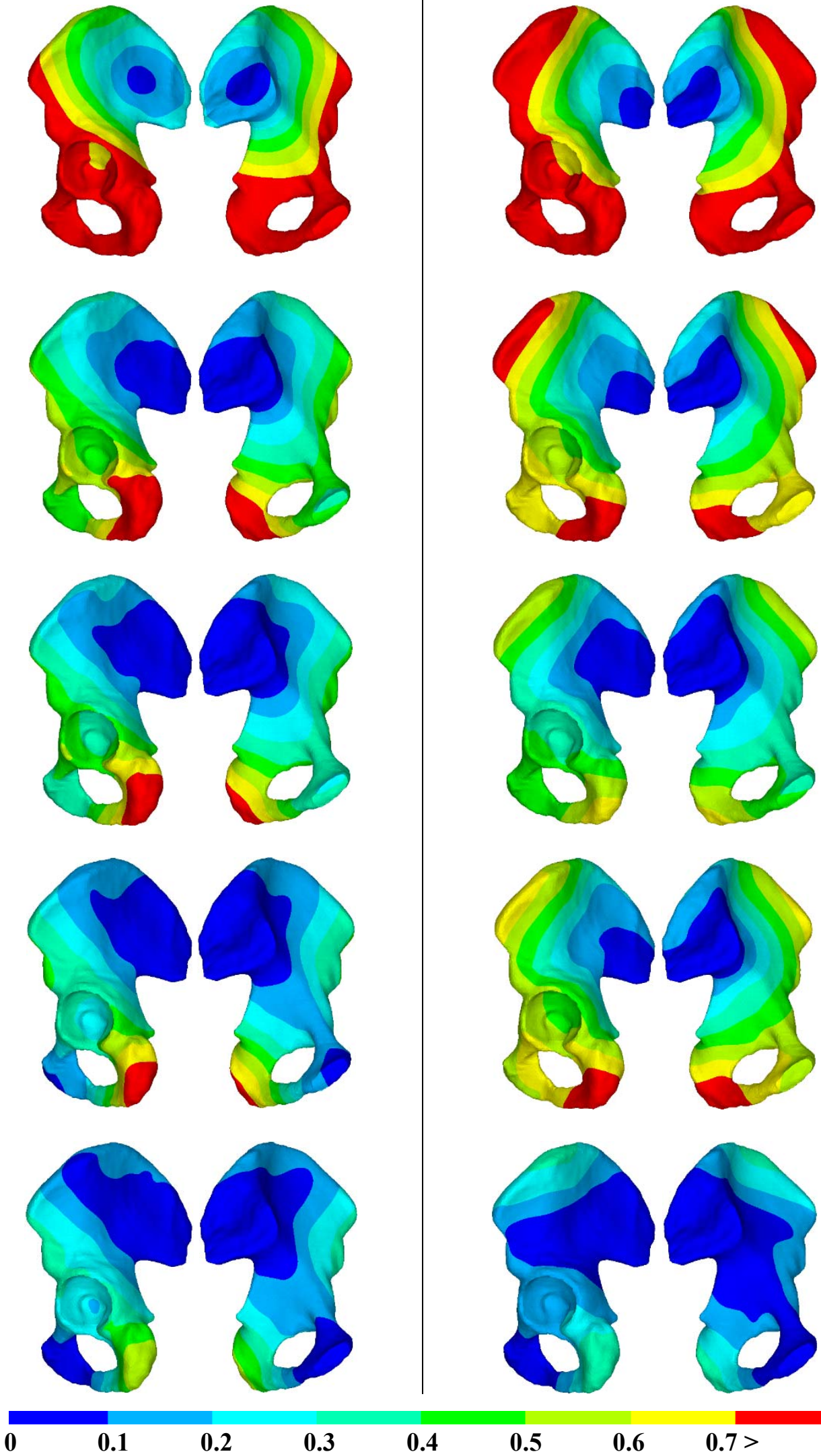

0.5

0.6

$0.7>$

Fig. 2. Displacement ( $\mathrm{mm})$ of the left hemi-pelvis under the two musculoskeletal loading regimes, showing: (a) Model 1; (b) Model 2; (c) Model 3; (d) Model 4; and (e) Model 5. 
(a)

(b)

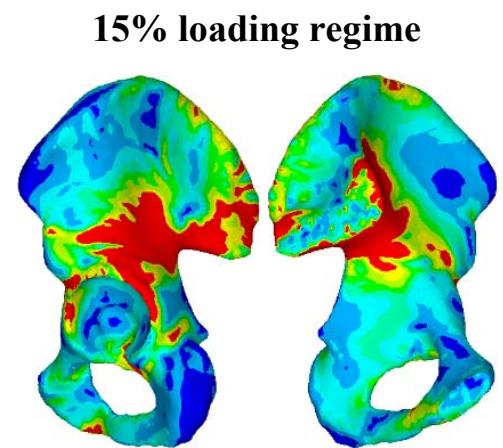

(c)

(d)
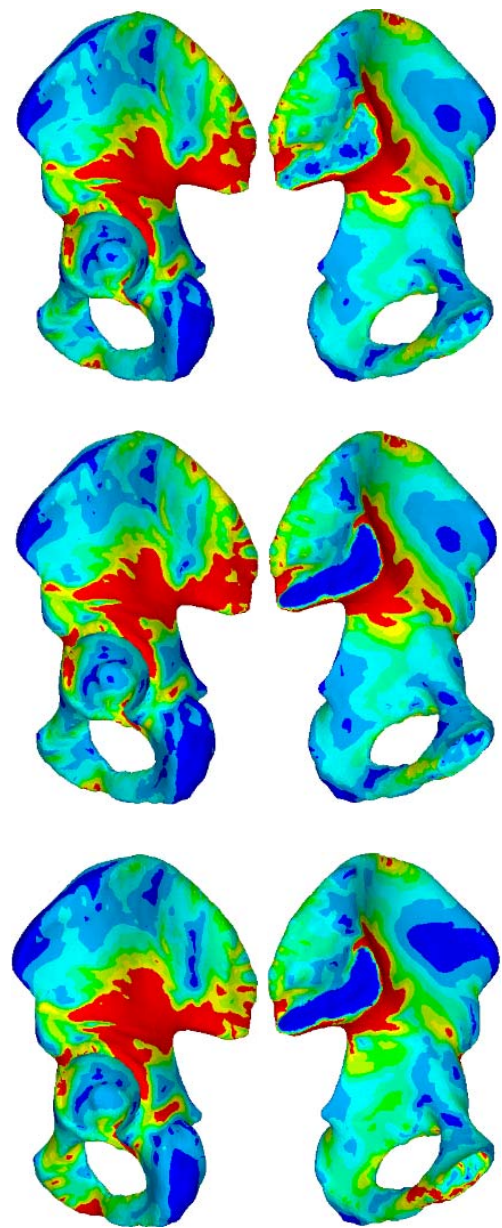

(e)
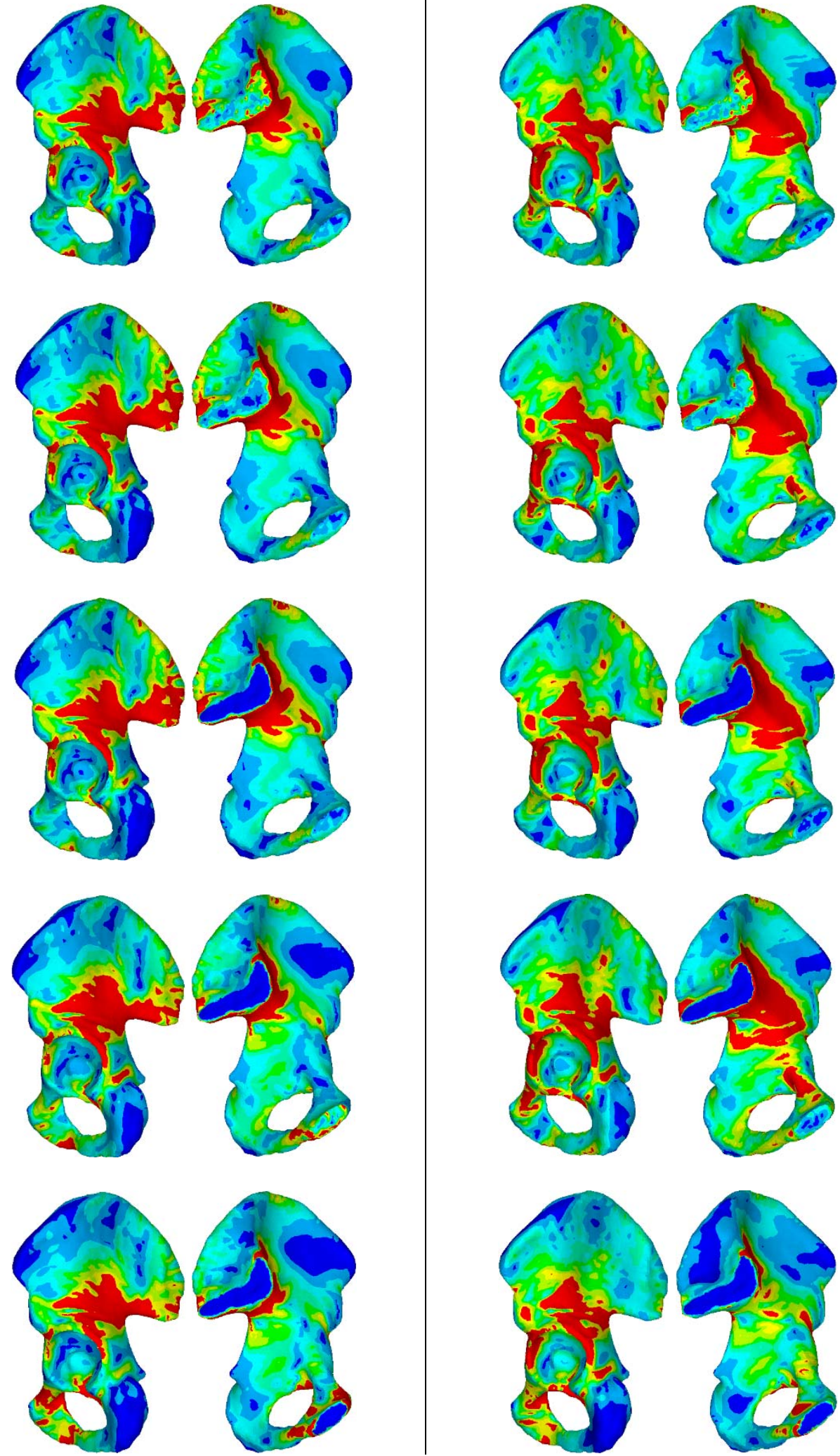

0
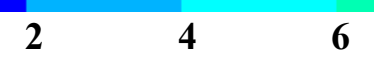

\section{8}
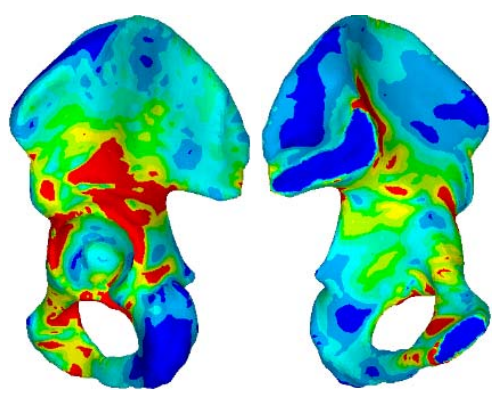

$14>$

Fig. 3. The von Mises stress distribution (MPa) of the left hemi-pelvis under the two musculoskeletal loading regimes, showing: (a) Model 1; (b) Model 2; (c) Model 3; (d) Model 4; and (e) Model 5. 


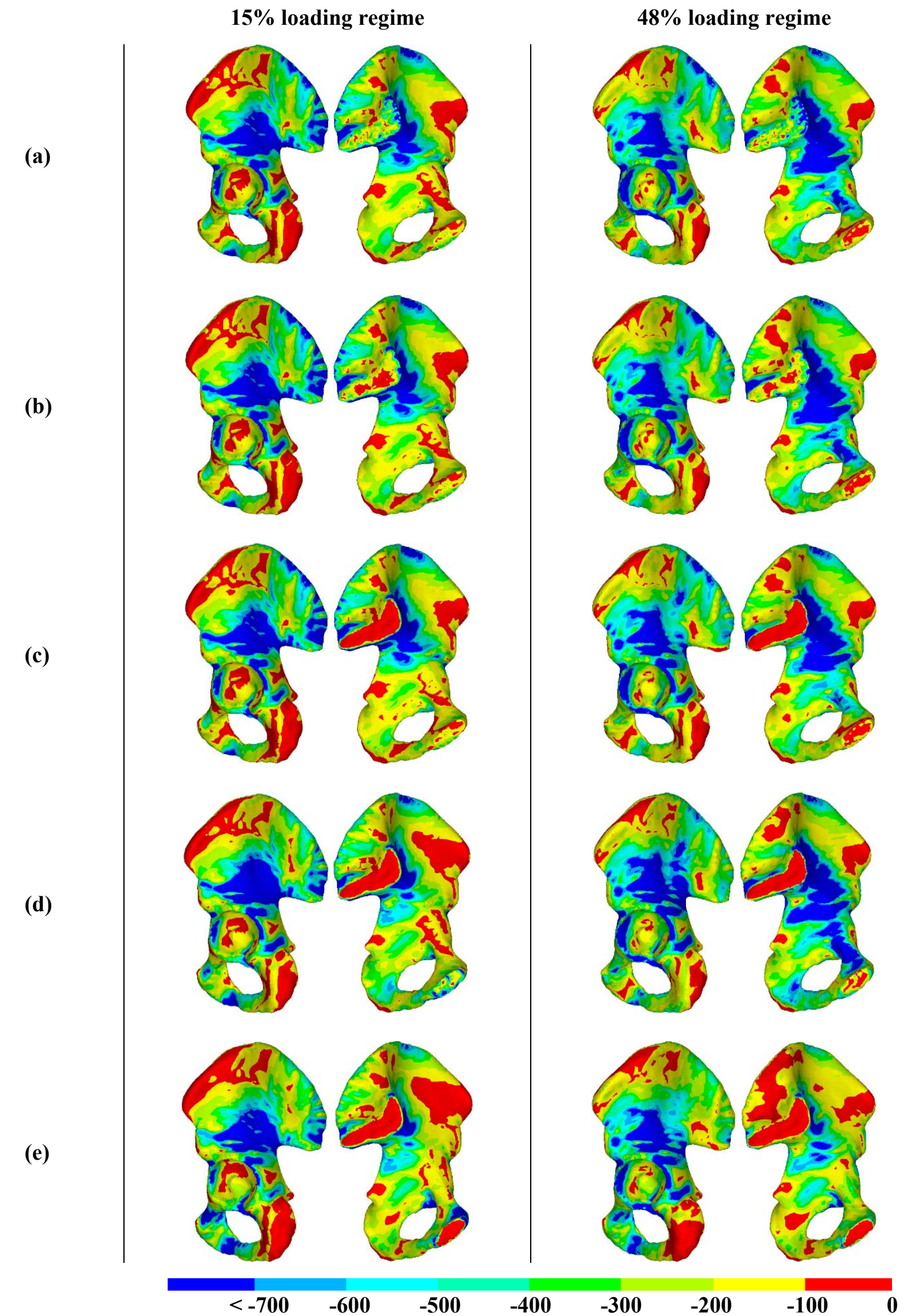

Fig. 4. The compressive strain distribution $(\mu \varepsilon)$ of the left hemi-pelvis under the two musculoskeletal loading regimes, showing: (a) Model 1; (b) Model 2; (c) Model 3; (d) Model 4; and (e) Model 5. 


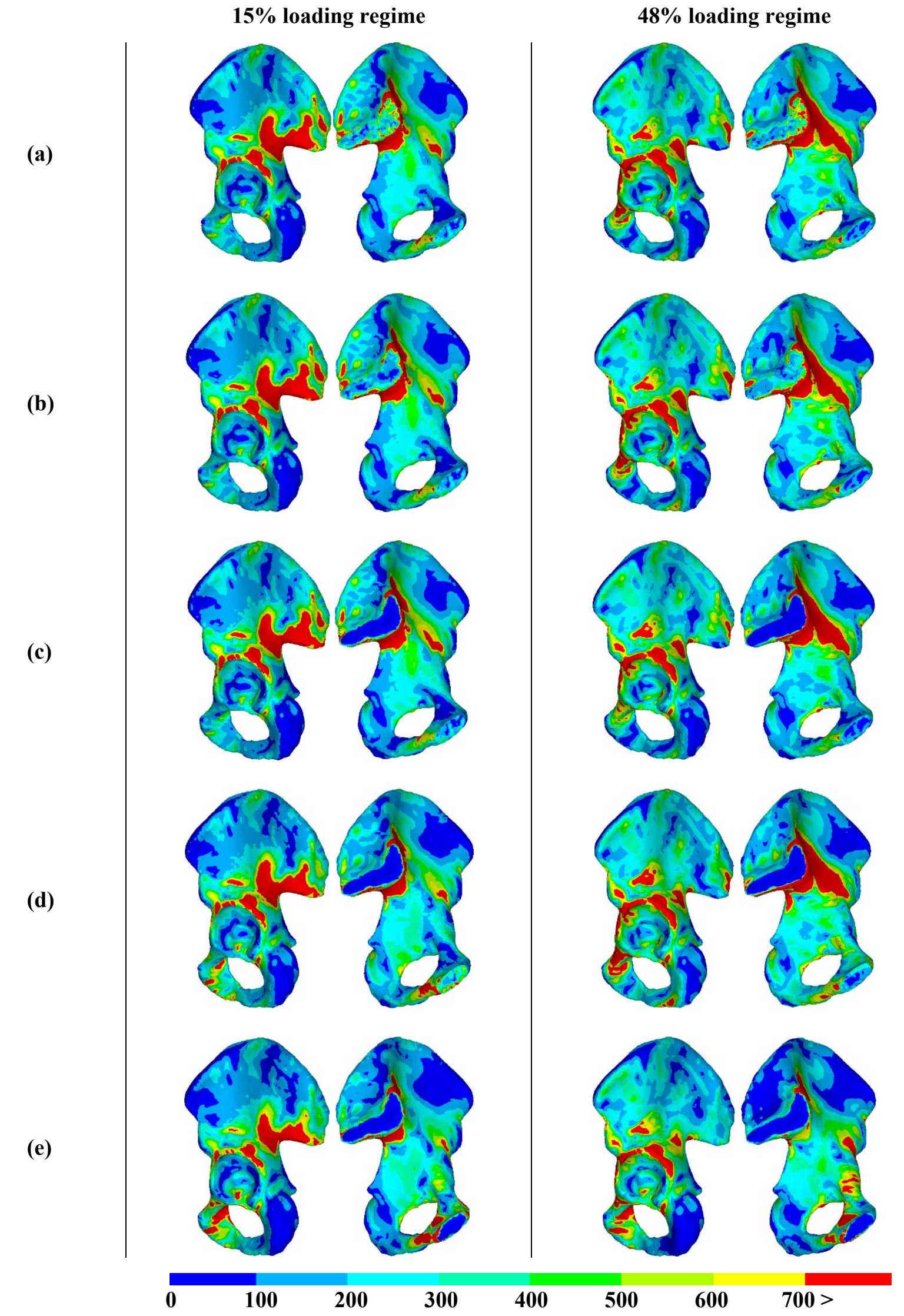

Fig. 5. The tensile strain distribution $(\mu \varepsilon)$ of the left hemi-pelvis under the two musculoskeletal loading regimes, showing: (a) Model 1; (b) Model 2; (c) Model 3; (d) Model 4; and (e) Model 5. 

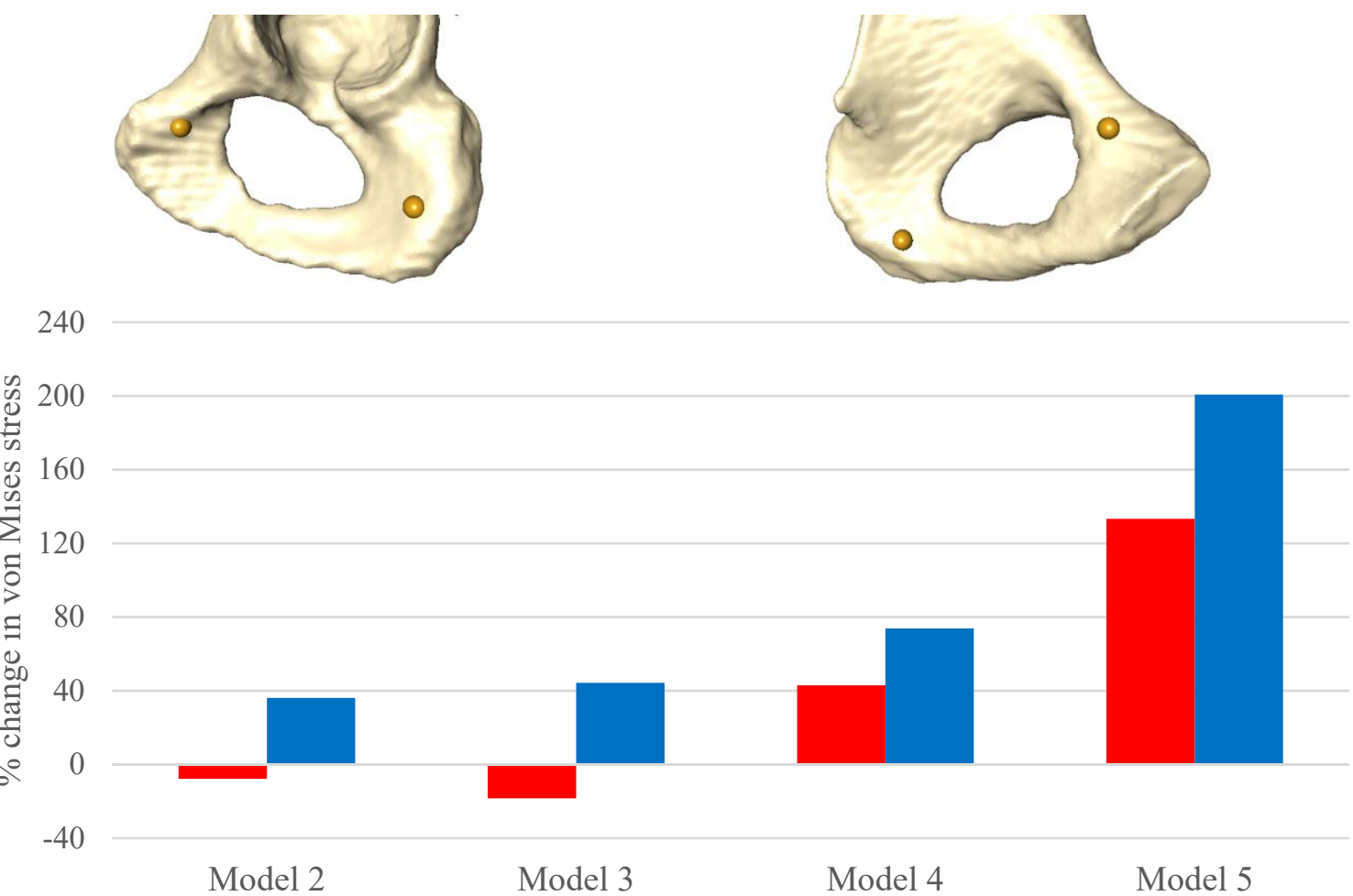

(a)

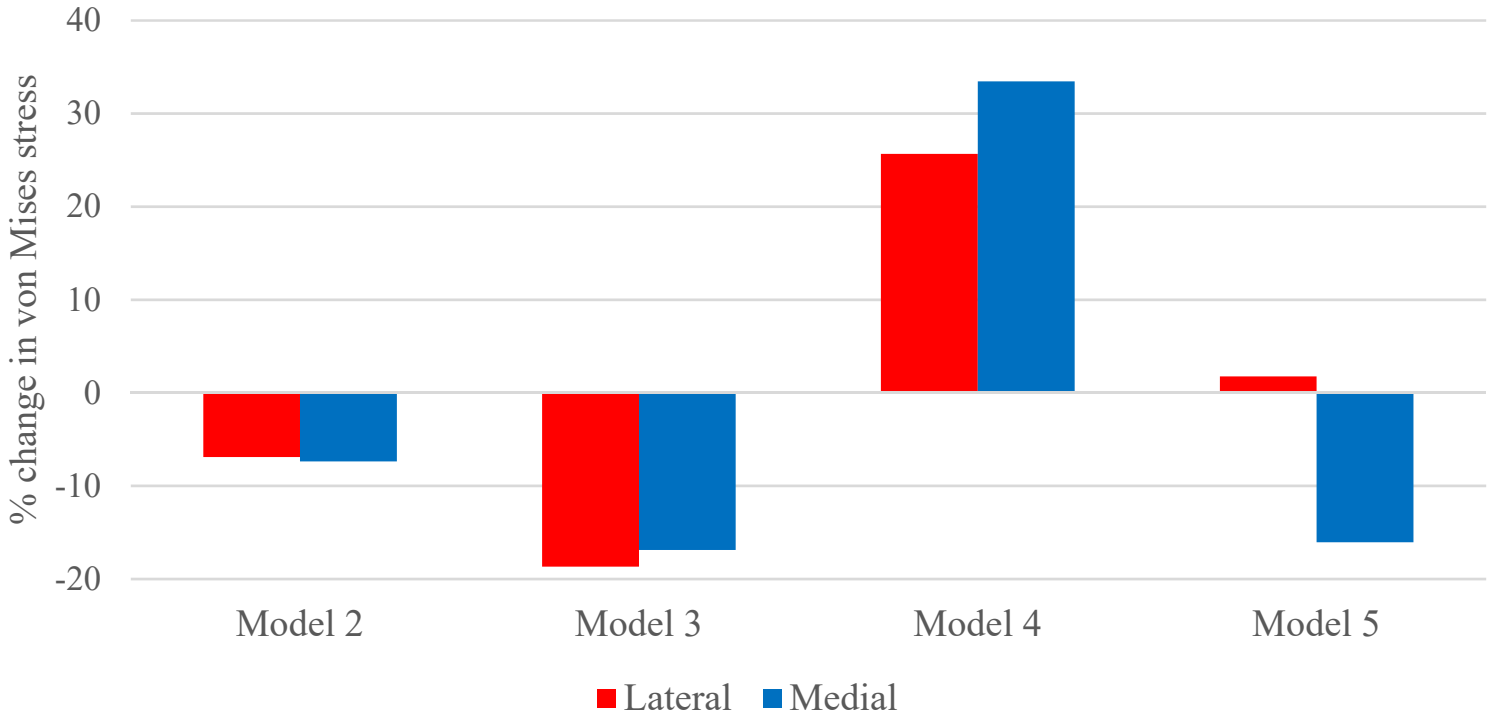

(b)

Fig. 6. The percentage change (with respect to Model 1) in the average nodal von Mises stress around a single location on the lateral and medial side of the (a) pubis and (b) ischium under the $15 \%$ loading regime. For comparison the nodal stresses in Model 1 were: (a) 8.02MPa in lateral and 1.52MPa in medial; (b) 2.51MPa in lateral and 5.79MPa in medial. The top images show the locations (shown as spheres) of the pubic and ischial sampling points. 


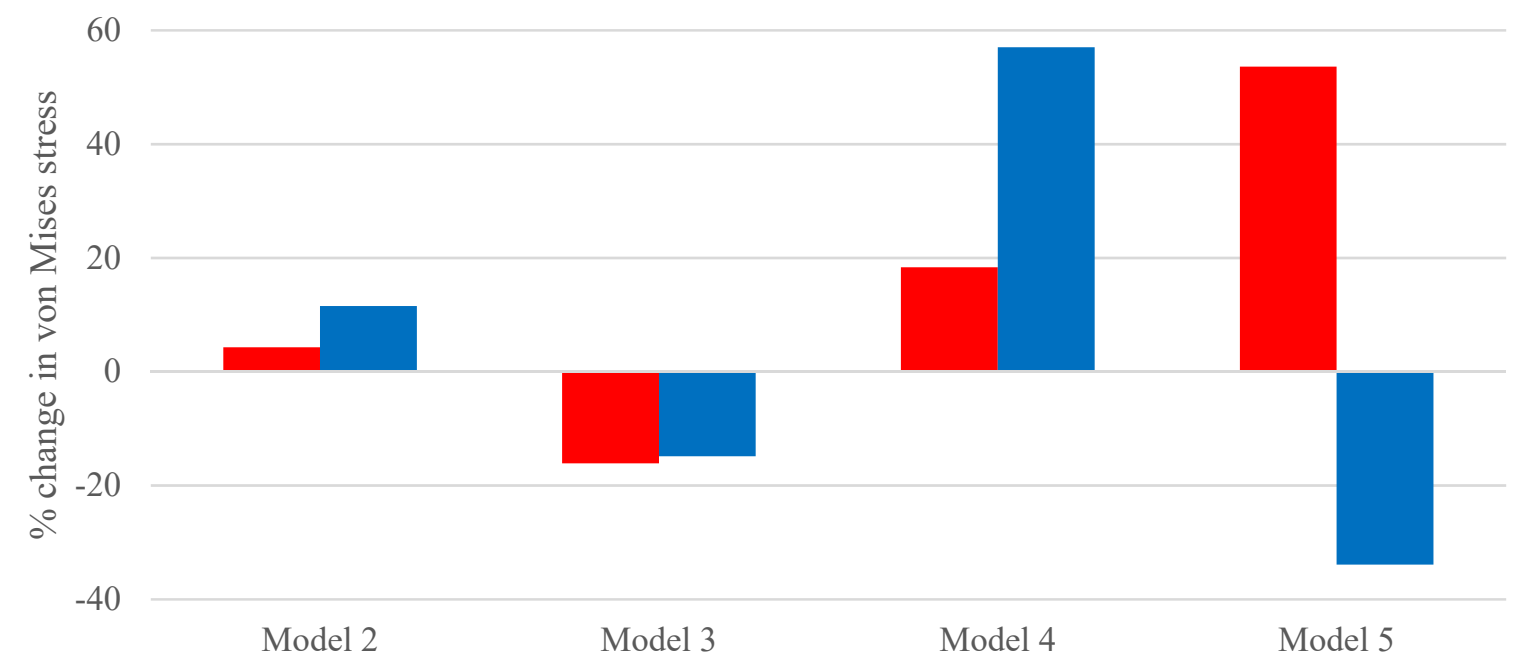

(a)

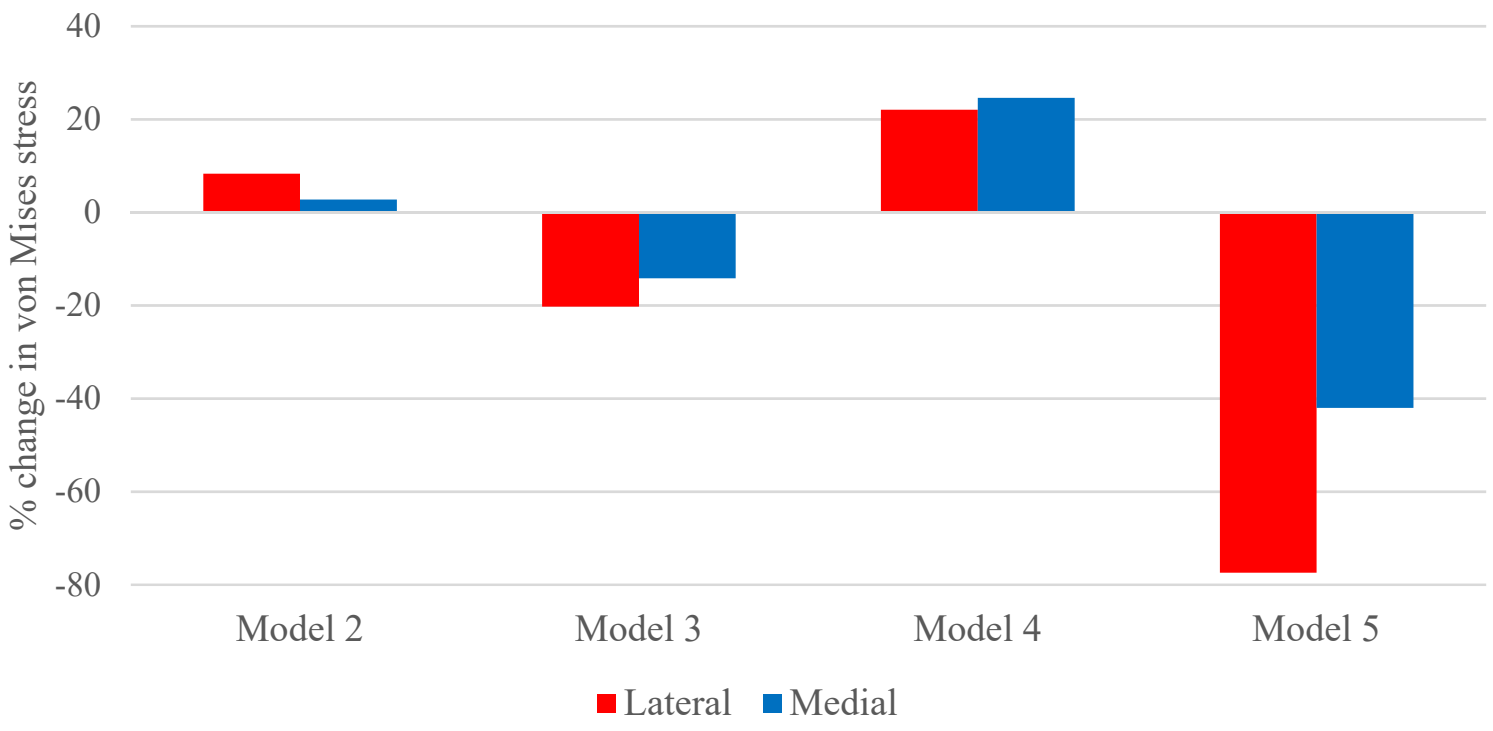

(b)

Fig. 7. The percentage change (with respect to Model 1) in the average nodal von Mises stress around a single location on the lateral and medial side of the (a) pubis and (b) ischium under the $48 \%$ loading regime. For comparison the nodal stresses in Model 1 were: (a) 11.86MPa in lateral and 11.62MPa in medial; (b) 4.54MPa in lateral and 7.54MPa in medial. 


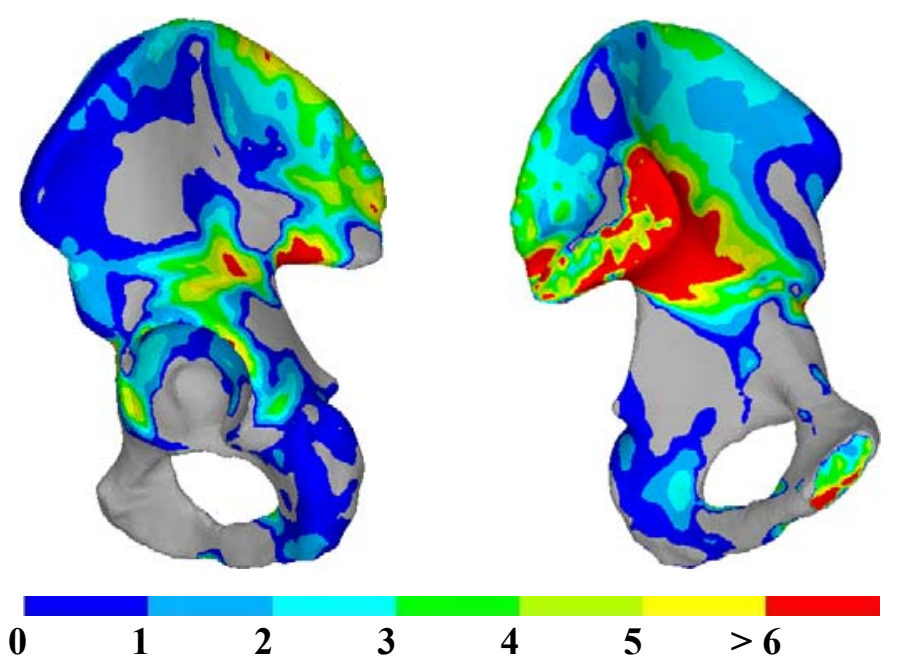

(a)

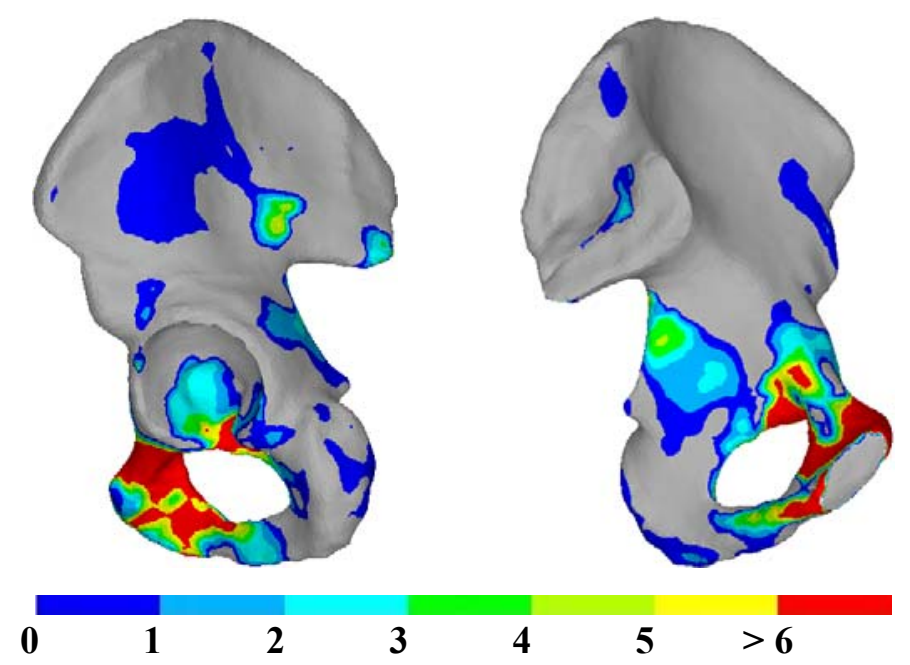

(b)

Fig. 8. Differences between the von Mises stresses (MPa) predicted in Models 1 and 5 under the $15 \%$ loading load regime. For eases of comparison, the contour plots display the regions where stresses are (a) higher in Model 1 and (b) higher in Model 5. The grey regions in each plot indicate where the stresses are lower in each case. 